cover the whole field of liver pathology the reviewer knows of no better account for the interested, whether pathologist, physician or surgeon.

The book contains an excellent account of acute infective hepatitis and of the new European classification of chronic hepatitis into chronic persistent hepatitis, often of little clinical consequence, and chronic aggressive hepatitis, which is so often associated with the clinical syndrome of active chronic hepatitis. Recent work on the pathogenesis of cirrhosis and the main histological division into macronodular and micronodular varieties is also clearly presented. The criteria given for the distinction of extra-hepatic biliary obstruction from intra-hepatic cholestasis due to drugs will be of considerable practical help.

There are useful accounts of staining techniques and an introductory chapter to electron microscopy. The black and white illustrations are particularly good and both author and publisher are to be congratulated.

\section{Biopsy Procedures in Clinical Medicine}

Ed. by A. E. ReAD. Pp. 208, illustrated. Bristol: John Wright \& Sons, 1968. £2.

This compact volume, ably edited by Dr Read, fills the long-felt need for a comprehensive reference book dealing with biopsy techniques in general medical practice. Contributors, mostly from the Bristol University School of Medicine, discuss techniques in their own fields-skin, kidney, marrow, lymph nodes, liver, spleen, gut, bone, synovia, lung, kidney, testis and ovary, with cytology and chromosome preparations discussed en route. Each chapter on a particular system gives the evolution of the relevant procedures, their indications at length, the necessary precautions and then the methods in detail, all with supporting references to the original literature with titles in full. Instruments and techniques are shown by diagram (probably more helpful than photographs in this regard). Although procedures are fully described, the importance of personal instruction by a master is repeatedly emphasised. A useful supplement shows where instruments may be obtained. An introductory chapter on laboratory procedures and staining methods illustrates the value of co-operation between clinician and pathologist for the best results.

In short, this is a book to be consulted on every medical ward and for the personal library of everyone interested in the craft of medicine. 